\title{
Implementation and Evaluation of Wireless Networked Control Systems using Modbus
}

\author{
M. R. Caldiéri, J. A. Bigheti and E. P. Godoy, Member, IEEE
}

\begin{abstract}
The recent introduction of wireless transmitters in the industry has driven a new interest in measuring and control techniques, but most applications are restricted to open loop monitoring applications. The reason is the lack of reliability due to problems inherent to the transmission medium, such as loss of information, time varying delays, slow and sometimes-aperiodic update measurements and interference. In order to enable and make the wireless networked control systems (WNCS) more reliable, many control techniques have been researched, including the PIDPlus representing a modified PID algorithm developed for use in systems in which the sampling frequency requirement cannot be guaranteed. This paper presents the implementation of the Modbus protocol for WNCS applications. The Modbus TCP was embedded in dedicated hardware enabling the transmission of data via Ethernet TCP/IP and Wi-Fi. A comparison of PID controllers for the WNCS subjected to variable delays and sampling, and packet losses were done. Experimental results in a pilot plant prove the efficiency of the implementation of a wireless control loop using a Wi-Fi network with embedded Modbus protocol and PIDPlus controller.
\end{abstract}

Keywords - PIDPlus, Wireless sensor, Modbus TCP/IP, Networked control.

\section{INTRODUÇÃO}

$\mathrm{O}$ CONCEITO de redes industriais fornece muitos benefícios, como uma maior flexibilidade e modularidade de instalações e a facilidade de configuração do sistema, comissionamento e manutenção [1]. As redes industriais podem ser divididas em três gerações distintas, com diferentes níveis de compatibilidade [2]. A primeira consiste em protocolos seriais fieldbus tradicionais, a segunda de protocolos baseados em Ethernet e a última geração, que começou a incorporar a tecnologia de comunicação sem fio (wireless).

Aplicações recentes de sistemas de controle distribuído demonstram o surgimento de uma nova abordagem para a utilização de redes industriais. Nessa abordagem, o controlador e a planta ficam fisicamente separados e são conectados por uma rede de comunicação. Este tipo de implementação em sistemas onde as malhas de controle são fechadas sob uma rede de comunicação industrial tem sido denominado de Sistema de Controle via Redes (NCS Networked Control System) [3].

Os sistemas de controle em rede possuem alguns problemas

\footnotetext{
M. R. Caldiéri, SENAI, Lençóis Paulista, SP, Brasil, mrcaldieri@gmail.com.

J. A. Bigheti, SENAI, Lençóis Paulista, SP, Brasil, jeferson.bigheti@sp.senai.br.

E. P. Godoy, Universidade Estadual Paulista (UNESP), Sorocaba, SP, Brasil.epgodoy@sorocaba.unesp.br

Corresponding author: Eduardo Paciencia Godoy.
}

inerentes a aplicações de controle que não existiam em sistemas analógicos e que são normalmente difíceis de serem detectados devido às variações e incertezas introduzidas pela rede de comunicação, como: atrasos, instabilidade, limitações de largura de banda e perda de pacotes [4]. Estas imperfeições e restrições relativas à rede industrial podem afetar significativamente o desempenho da malha de controle, podendo até torná-lo instável.

Avanços recentes na tecnologia de redes de sensores sem fio levaram ao desenvolvimento de dispositivos de baixo custo e baixo consumo de energia. Com esses avanços, uma nova tendência surgiu com o uso de redes sem fio em NCS [5], promovendo interoperabilidade entre redes com fio já existentes e novas redes sem fio. Estes sistemas são conhecidos como sistemas de controle via redes sem fio (WNCS - Wireless Networked Control Systems). WNCSs ultimamente têm atraído esforços de pesquisa e desenvolvimento, relacionados à crescente evolução e padronização de redes sem fio como ZigBee, Wi-Fi [6], Wireless Hart e ISA-100.11a [7].

Muitas características interessantes inerentes às redes sem fio estão motivando o desenvolvimento de WNCS. Os sistemas sem fio proporcionam vantagens [1] como a redução na quantidade de fiação necessária para a comunicação, que por sua vez reduz os custos de instalação, comissionamento e reconfiguração pode ser realizada mais rápido, instalação preferencial em equipamentos que se movimentam onde o cabeamento pode ser facilmente danificado, em ambientes perigosos e instalações de segurança intrínseca, em localidades onde cabos pode restringir o funcionamento das máquinas a serem monitoradas e em ambientes com longas distâncias entre dispositivos e de difícil acesso como em refinarias ou de outras plantas de processamento. O WNCS pode ser operado entre os sistemas com fio e sem fio existentes e também proporciona vantagens em relação a potência e flexibilidade quando comparados aos com fio formando uma rede híbrida [8].

Esta introdução de dispositivos sem fio na indústria de processo provocou um novo interesse em técnicas que podem ser usadas para permitir que o controle em malha fechada, seja usado com atualizações de informação não periódicas [9]. Um pressuposto de controle de processo sempre foi que o controle é executado em uma base periódica e que um novo valor de medição está disponível para cada execução. No entanto, para minimizar o consumo de energia de um transmissor sem fio, os valores de medição podem ser transmitidos com uma frequência baixa, ou somente se o valor da medição mudar significativamente [9]. Desta forma o controle tem que ser modificado para trabalhar com atualizações não periódicas de 
medição. Além disso, é importante que a perda de comunicação seja tratada automaticamente pelo controle de uma forma a não introduzir uma interrupção do processo.

Quando a medição não é atualizada em uma base periódica, um PID tradicional não é indicado, pois as ações de controle não serão calculadas de forma correta. Se o controle só é executado quando uma nova medida é informada, isso poderia resultar em um atraso na resposta de um controlador. Assim, um desafio tem sido a proposta de uma técnica que minimize a frequência de medição de uma variável sem comprometer o desempenho do controle. Para resolver estas questões o algoritmo PID pode ser modificado para funcionar corretamente com atualizações de medição lentas, não periódica e perda de comunicação, como no caso do PIDPlus que representa um controlador em malha fechada usando um transmissor de rede sem fio [9].

Este artigo está apresentado da seguinte forma. A seção II apresenta uma revisão sobre as redes de comunicação usadas. A implementação do protocolo Modbus e uma descrição do WNCS desenvolvido na planta piloto do SENAI é mostrado na secção III. A seção IV discute os experimentos realizados e os resultados obtidos para o WNCS. A seção V elenca as conclusões e contribuições deste artigo.

\section{REDES DE COMUNICAÇÃO}

O Modbus é um protocolo aberto que define uma estrutura de mensagens de comunicação usadas para transferir dados discretos e analógicos entre dispositivos microprocessados com deteç̧ão e informação de erros de transmissão. A camada de aplicação Modbus define uma unidade de protocolo de dados simples, independente das camadas inferiores (PDU) mostrada na Fig. 1. O mapeamento do protocolo em portas específicas ou redes se dá por meio da unidade de aplicação de dados (ADU).

\section{General MODBUS Frame}

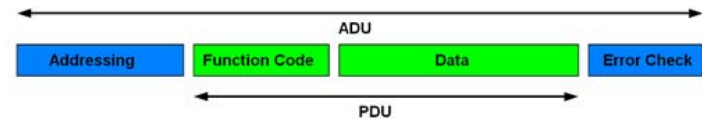

Figura 1. Estrutura de uma Mensagem Modbus [10].

O protocolo Modbus é implementado em três versões [10]:

1. Modbus TCP/IP - os dados são encapsulados em formato binário, em quadros TCP (Transmission Control Protocol), usando o protocolo IP e Ethernet (IEEE 802.3). O controle de acesso ao meio utilizado é o Acesso Múltiplo por Detecção de Portadora com Deteç̧ão de Colisão (CSMACD);

2. Modbus Serial - opera em meio físicos variados (fios, fibra óptica e rádio). Os principais protocolos são: EIA / TIA232-E (conhecida como RS232), EIA-422 (conhecida como RS422) e EIA / TIA-485-A (conhecida como RS485). O modo de transmissão em serial tem duas variantes: Modbus RTU os dados são transmitidos em formato binário 8-bit e Modbus ASCII - os dados são transmitidos em formato ASCII de 7 bits;

3. Modbus Plus - rede de transferência de alta velocidade com recursos adicionais para o encaminhamento, $\mathrm{o}$ diagnóstico, a consistência dos dados e endereçamento. Embora mais robusto e eficiente, este modo não tem uma especificação aberta, uma vez que é de domínio da Schneider Electric.

Modbus TCP/IP é simplesmente o protocolo Modbus com uma interface Ethernet TCP/IP. O Modbus TCP/IP utiliza o TCP/IP e Ethernet para transportar os dados da estrutura de mensagem Modbus entre dispositivos compatíveis. Isto é, o Modbus TCP/IP combina uma rede física (Ethernet), com um protocolo de rede (IP) e transporte (TCP), e um método de representação de dados padrão (Modbus como o protocolo da aplicação). A principal função do TCP é garantir que todos os pacotes de dados são recebidos corretamente, enquanto IP garante que as mensagens são corretamente endereçadas $\mathrm{e}$ encaminhadas. Conforme mostrado na Fig. 2, o encapsulamento TCP/IP não modifica a estrutura básica da mensagem Modbus. As diferenças estão na interpretação do endereço (IP) e na verificação de erro (CRC-32).

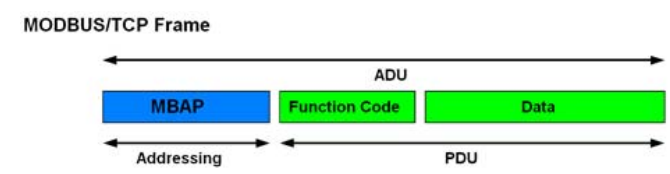

Figura 2. Estrutura de uma Mensagem Modbus TCP/IP [10].

Redes locais sem fio WLAN podem operar em 2,4 GHz ou $5 \mathrm{GHz}$. Estas redes suportam dois modos: ad-hoc e de infraestrutura. O modo ad-hoc permite que todas as estações se comunicam uns com os outros de forma peer-to-peer. O modo de infraestrutura, a rede tem um ponto de acesso (AP), por meio do qual cada estação cliente se comunica.

As vantagens de WLAN em automação de processos incluem: padrões abertos, robustez, boa relação custobenefício, fácil acessibilidade e alta taxa de transmissão de dados. A segurança também aumentou com duas melhorias de segurança, Wi-Fi Protected Access (WPA) e WPA2, que substituem tecnologia mais antiga e menos segura.

O protocolo Wi-Fi IEEE 802.11 possui uma característica importante entre os dispositivos sem fio que deve ser considerada quando o controle é implementado através de uma WLAN. Embora o protocolo IEEE 802.11, não tenha sido concebido para aplicações em WNCS, a sua implementação pode ser útil no controle de rede quando define o DCF (Distributed Control Function). Neste protocolo, a decisão da estação que pode transmitir é feita entre os nós da WLAN [11].

\section{MATERIAIS E MÉTODOS}

Modbus é um protocolo de comunicação da camada de aplicação, representada pela camada sete do modelo OSI de camadas e, portanto, independente das outras seis camadas, sendo apenas necessário que elas sejam funcionais. Isso permite vários métodos de implementar o Modbus com diferentes meios físicos. Neste trabalho, foram implementadas uma versão do Modbus TCP/IP e do Modbus Wi-Fi, baseadas em Arduino, para uso em aplicação de WNCS, conforme mostrado na Fig. 3. 


\begin{tabular}{|c|c|c|c|}
\hline \multicolumn{2}{|c|}{ Camadas ISO/OSI } & Modbus TCP/IP / Wi-Fi & Implementação \\
\hline 7 & Aplicação & Modbus & Arduino \\
\hline 6 & Apresentação & & \\
\hline 5 & Sessão & & \\
\hline 4 & Transporte & Protocolo TCP & Pilho TCP/PP \\
\hline 3 & Rede & Protocolo IP & 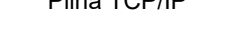 \\
\hline 2 & Enlace & We: & Ethernet Shield ou \\
\hline 1 & Física & Etnernet ou vai-ri & Módulo XBee Wi-Fi \\
\hline
\end{tabular}

Figura 3. Implementação do Modbus.

A camada de aplicação do Modbus foi implementada em código na plataforma Arduino. Através do uso de uma pilha TCP/IP compatível, juntamente com um módulo shield Ethernet e um módulo XBee WiFi é possível realizar a comunicação de dados via Modbus TCP/IP e Modbus Wi-Fi. Através da integração dos equipamentos citados, é possível a comunicação com e sem fio utilizando os protocolos Modbus e TCP/IP. No protocolo Modbus TCP/IP, os dados do frame Modbus são transmitidos através do protocolo TCP, juntamente com um endereço IP, de acordo com o modelo Cliente/Servidor de comunicação. O protocolo TCP deve estabelecer uma conexão antes de transferir os dados, uma vez que é um protocolo baseado em conexão ponto a ponto. $\mathrm{O}$ Mestre/Cliente Modbus estabelece uma conexão com o Escravo/Servidor. O Servidor aguarda por uma conexão de entrada do Cliente, e estabelecida a conexão, responde às consultas ou requisições de dados até que a conexão seja encerrada.

A implementação do Modbus TCP/IP foi realizada utilizando um microcontrolador Arduino juntamente com um Ethernet Shield e com um transmissor sem fio Wi-Fi (XBee S6B). Este conjunto representa o Servidor Modbus, o qual é parametrizado com um endereço IP. O Servidor não tem uma identificação, sendo que todo endereçamento da comunicação é baseado no endereço IP. Ao receber a solicitação de informação do Cliente da rede (controlador da malha de controle em LabVIEW), o XBee irá repassar a solicitação ao Arduino de forma serial no protocolo Modbus. A solicitação do Cliente Modbus é salva numa matriz do Arduino para interpretação de cada byte da mensagem. Através do código de função Modbus recebido (código 04: relativo à solicitação de leitura de registrador analógico de 16bits), é possível responder ao Cliente com a informação requerida (entrada analógica que está ligada ao pino 13 do Arduino) armazenada no registrador solicitado (registrador 40010).

É importante verificar que existem diferenças no conteúdo da mensagem Modbus serial (RTU) e a Modbus TCP/IP, conforme apresentado na Fig. 4. Essa lógica de montagem e interpretação dos frames Modbus para viabilizar a comunicação é realizada pela Arduino.
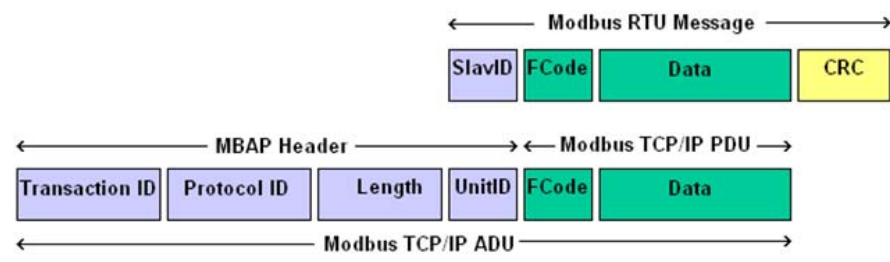

Figura 4. Diferença entre mensagem Modbus RTU e Modbus TCP/IP [10].
A introdução de sensores sem fio na indústria origina novos desafios quando a medição da variável é utilizada em aplicações de controle. Para lidar estes desafios, o controlador PID pode ser modificado para operar de forma adequada com grandes períodos de amostragem do sensor, atualizações não periódicas da variável e perda de mensagens transmitidas [9]. A chave para se compreender como o PID deve ser modificado é realizando o reset do PID implementado utilizando uma rede de realimentação e um filtro onde a constante de tempo é um reflexo direto da resposta dinâmica do processo.

A implementação PIDPlus [12] para WNCS é ilustrada na Fig. 5. O PIDPlus mantém o sinal de controle no último nível calculado até que uma nova medida seja recebida. É importante notar que sua sintonia é independente do período de amostragem, depende apenas das características físicas da planta.

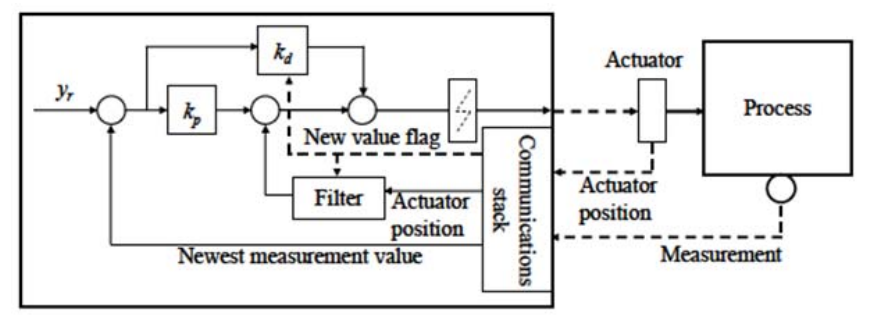

Figura 5. Estrutura do controlador PIDPlus [12].

A realimentação (newest measurement value) e o filtro de $1^{\mathrm{a}}$ ordem (filter) são modificados para criar a contribuição de reposição com o seguinte comportamento:

$\checkmark$ manter a última saída do filtro calculado $\left(\mathrm{F}_{\mathrm{N}-1}\right)$, até uma nova medição ser informada (new value flag);

$\checkmark$ quando uma nova medição é recebida (new value flag), utilize a nova saída do filtro como contribuição da realimentação $\left(\mathrm{F}_{\mathrm{N}}\right)$.

A principal diferença do PID e PIDPlus está na parte integrativa que foi substituído por um filtro de $1^{\mathrm{a}}$ ordem. A saída do filtro é calculada conforme (1):

$F_{N}=F_{N-1}+\left(O_{N-1}-F_{N-1}\right)\left(1-e^{\frac{-\Delta T}{T_{\text {reset }}}}\right)$

Onde: $F_{N}=$ nova saída do filtro, $F_{N-1}=$ saída do filtro na ultima execução, $O_{N-1}=$ saída do controlador na ultima execução e $\Delta T=$ intervalo de tempo desde que o último valor medido foi recebido e $\mathrm{T}_{\text {reset }}=$ constante de tempo da planta somado ao tempo morto.

A parte derivativa é substituída conforme (2):

$O_{D}=K_{D} \frac{e_{N}-e_{N-1}}{\Delta T}$

Onde: $e_{N}=$ erro atual, $e_{N-1}=$ ultimo erro, $O_{D}=$ termo derivativo do controlador e $K_{D}=$ ganho derivativo.

Considere a contribuição da parte derivativa quando as entradas são perdidas durante vários períodos. Para $\mathrm{o}$ algoritmo PID tradicional, o divisor na parte derivativa seria o período (discretização do controlador), enquanto que, no 
algoritmo PIDPlus é o tempo decorrido entre duas medições recebidas com sucesso $(\Delta \mathrm{T})$. É óbvio que o algoritmo modificado produz uma ação derivativa menor do que o algoritmo de controle PID [12]. Na implementação do PIDPlus, o cálculo de reposição compensa automaticamente a alteração da medição e taxa de atualização da medição. Os cálculos do termo derivativo para um novo valor de medição não estão disponíveis a cada execução do PID. Assim, não há necessidade de modificar a sincronização para o controle sem fio, ou seja, o ajuste é baseado estritamente no ganho e dinâmica do processo.

A implementação do sistema de controle via rede sem fio (WNCS) em estudo, foi baseada em uma Planta Piloto de Instrumentação da escola SENAI de Lençóis Paulista mostrada na Fig. 6, da qual foi usada uma malha de controle de vazão.

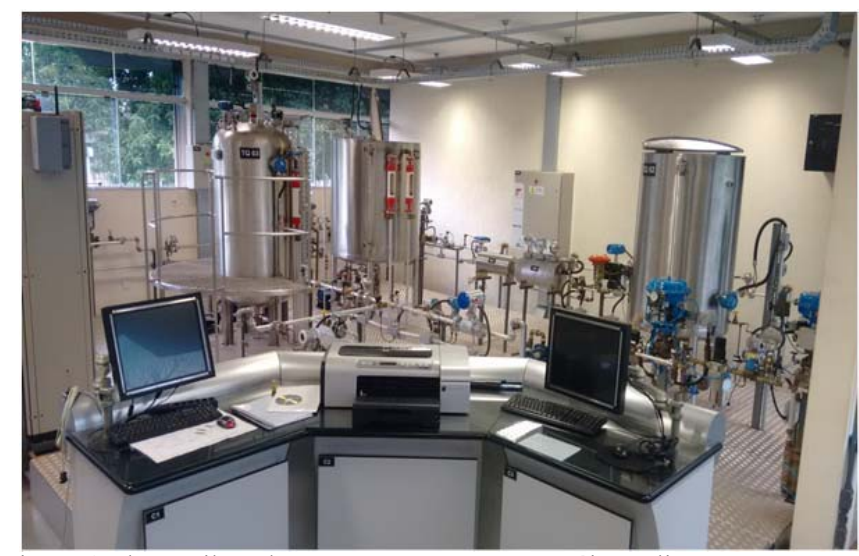

Figura 6. Planta Piloto de Processos - SENAI Lençóis Paulista.

O esquemático do WNCS é mostrado na Fig. 7. Em um medidor de vazão magnético desta planta foi instalado um conversor de sinal analógico (0 a 10Vcc) para $\mathrm{Wi}-\mathrm{Fi}$, com o pacote Modbus na camada de aplicação, onde foi criada uma rede sem fio entre o medidor de vazão, um Access Point e um Notebook (controlador).

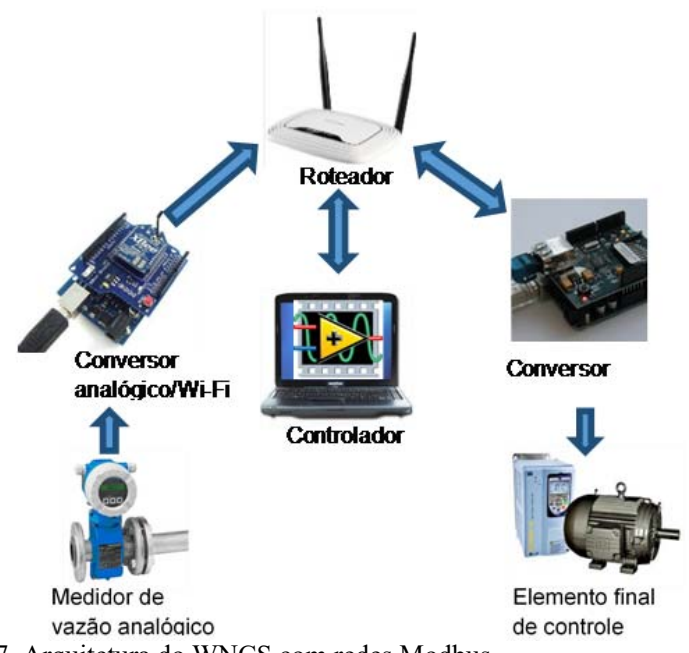

Figura 7. Arquitetura do WNCS com redes Modbus.

O sinal de controle é transmitido através de uma rede Ethernet TCP/IP entre o Access Point e um conversor de Ethernet para sinal analógico $(0$ a $5 \mathrm{Vcc})$ para um inversor de frequência que está ligada a uma moto-bomba que fará o controle da vazão. Para o cálculo do sinal de controle, foram implementados um controlador PID tradicional e um PIDPlus. O período de amostragem do sensor sem fio e atualização do controlador usada foi de $2 \mathrm{~s}$.

\section{RESULTADOS E ANÁLISE}

Os principais parâmetros relacionados ao desempenho de WNCS são o atraso de comunicação, jitter e a perda de mensagens transmitidas. O mais crítico destas métricas é o tempo que decorre entre o envio de um dado de um nó até o outro recebê-lo ou o tempo de atraso [13].

Uma maneira de medir esse tempo de atraso é medir o tempo de comunicação de ida e volta dentro da malha. O tempo de viagem é o tempo de atraso no envio de um pacote de um nó para outro e vice-versa. Se um cálculo estatístico é realizado a partir de uma quantidade de atrasos, podemos obter o tempo de atraso médio (Td) das transmissões de mensagens na rede sem fio. A variabilidade nos valores de medição do tempo de atraso fornece o Jitter (J) do WNCS como podemos ver na Fig. 8.

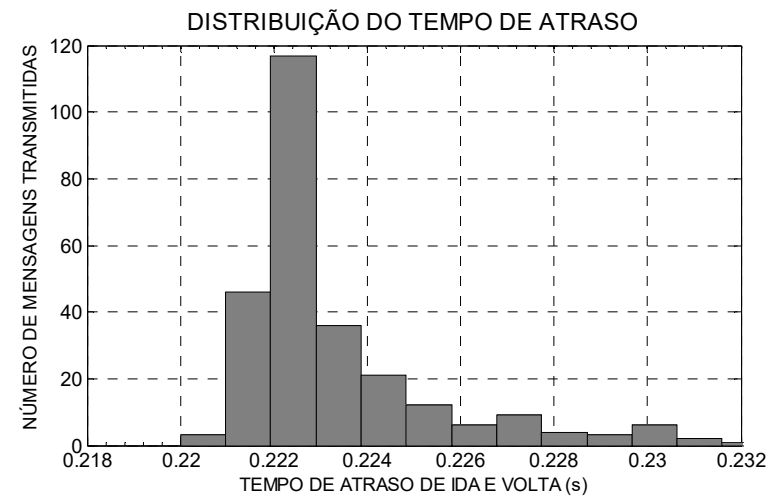

Figura 8. Histograma do tempo de atraso da rede Modbus Wi-Fi.

A Fig. 8 apresenta a distribuição dos tempos de atraso obtidos com o envio de mensagens no Modbus Wi-Fi. Como pode ser observado, a maioria dos valores aproximam-se do valor médio de $0,2226 \mathrm{~s}$. Sem a presença de grandes valores extremos, o histograma nos mostra um bom determinismo, sem perda de mensagens nos testes realizados, como é possível observar na Tabela I.

TABELA I

DESEMPENHO DA REDE SEM FIO NO CONTROLE PID

\begin{tabular}{|c|c|c|c|c|}
\hline Controle & Td $(\mathbf{m s})$ & $\mathbf{J}(\mathbf{m s})$ & Pior valor $(\mathbf{m s})$ & Mensagem Perdida \\
\hline PID & 222,6 & 10,7 & 231 & 0 \\
\hline
\end{tabular}

Os controladores PID e PIDPlus foram submetidos às mesmas condições de operação e distúrbios para que as respostas possam ser analisadas de forma qualitativa $\mathrm{e}$ quantitativa. O comportamento dos controladores para o WNCS de vazão em situações normais, ou seja, com a transmissão de dados sem fio eficiente e sem interferências ou erros, somente variação de setpoint (SP) foi usado para comparação de desempenho. Conforme mostrado na Fig. 9, os desempenhos de controle do WNCS em operação livre de erros são semelhantes para o controlador PID e PIDPlus. 


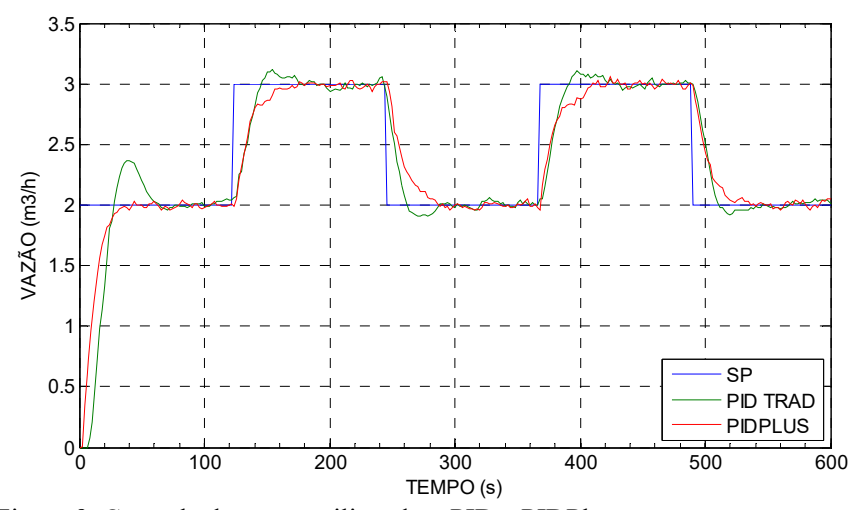

Figura 9. Controle de vazão utilizando o PID e PIDPlus.

Para avaliar uma situação real de operação do WNCS, foram inseridas falhas de comunicação (perda de mensagens transmitidas pelo sensor sem fio), onde a cada $60 \mathrm{~s}$ de operação eram gerados $5 \mathrm{~s}$ de falha $(60 \times 5)$. Nota-se, na Fig. 10 , que nos momentos de falha, o processo se desestabilizou pelo fato do controlador não se adequar à ausência da realimentação, causando a saturação da saída do controlador e um desgaste excessivo no elemento final de controle. Em um processo industrial, essa situação seria prejudicial e deveria ser evitada. Na Fig. 10 podemos observar as falhas de comunicação da variável do processo onde não há marcadores no gráfico da resposta do WNCS.

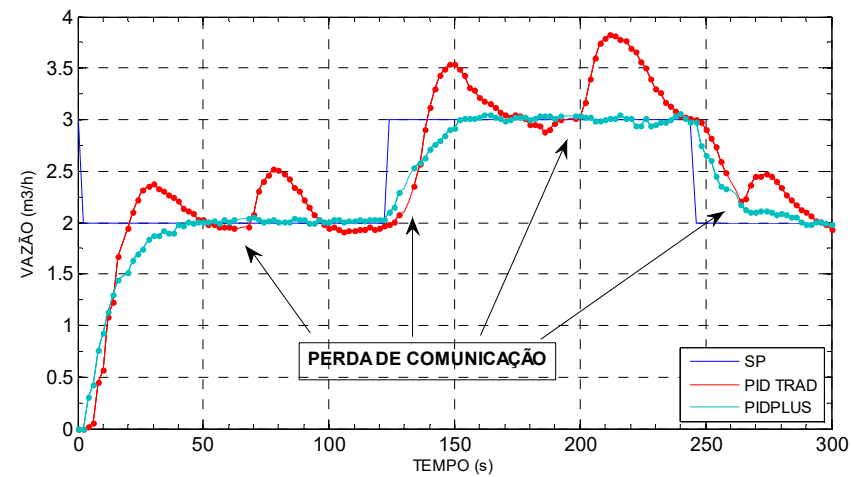

Figura 10. WNCS de vazão: PID e PIDPlus com falhas de comunicação.

$\mathrm{Na}$ Tabela II é possível comprovar que o desempenho de controle do PIDPlus para o WNCS operando sob falhas, é melhor que o PID tradicional.

Para verificar a robustez do WNCS com o PIDPlus, foram realizados testes adicionais comparando os controladores, para o mesmo perfil de distúrbios e com uma maior frequência de falhas de comunicação: $30 \mathrm{~s}$ de operação e $6 \mathrm{~s}$ com falha na comunicação $(30 \times 6)$ e com $15 \mathrm{~s}$ de operação e $6 \mathrm{~s}$ com falha (15x6). Lembrando que o período de amostragem é de $2 \mathrm{~s}$, portanto três mensagens são perdidas. Os resultados dessa comparação são mostrados na Fig. 11.

Na Fig. 11, é possível verificar notória degradação no desempenho de controle com o PID tradicional, ocasionando uma resposta muito oscilatória, já que o tempo (entre falhas) que o controlador tinha para se ajustar diminuiu. Este controlador nestas condições não poderia ser utilizado neste processo. Em contrapartida, o PIDPlus obteve desempenho melhor, controlando o WNCS adequadamente.

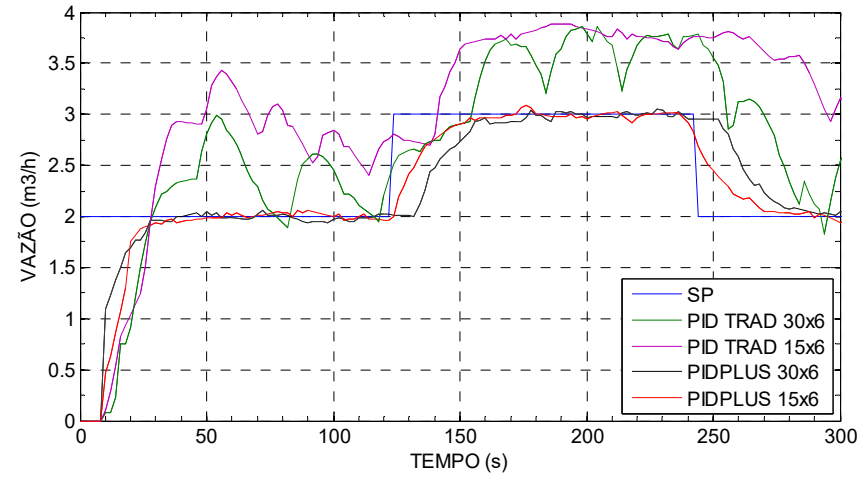

Figura 11. Comparação do WNCS de vazão com maior frequência de falhas de comunicação.

Também foi realizado uma análise do desempenho do WNCS operando com PIDPlus na presença de falhas de comunicação randômicas. Nesse caso, somente foi analisado o PIDPlus pela impossibilidade de replicar o mesmo experimento para o PID tradicional. Nesse teste, mostrado na Fig. 12, as falhas são variáveis de 2 a $10 \mathrm{~s}$ e ocorrem aleatoriamente em qualquer instante de operação.

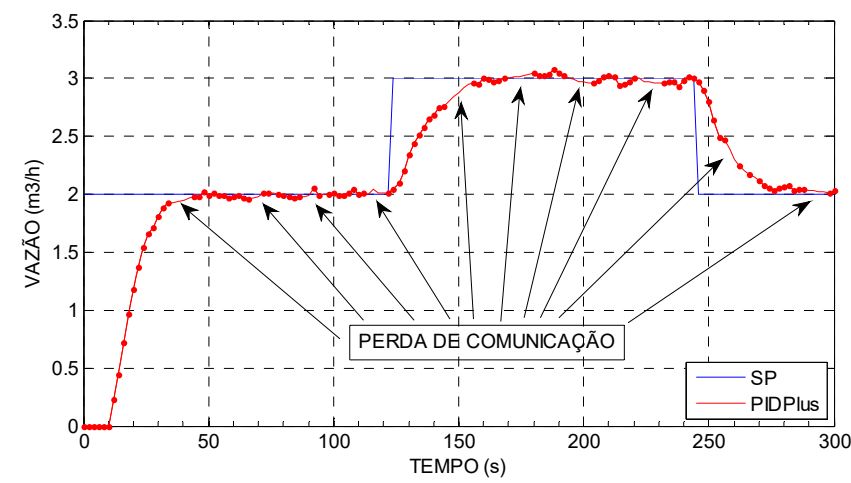

Figura 12. WNCS de vazão: PIDPlus com falhas de comunicação aleatórias.

A Tabela II apresenta um comparativo de todos os resultados dos índices de desempenho de controle baseados no erro da resposta (IAE e ITAE) para os testes de operação do WNCS de vazão com os controladores. Observa-se pelos valores dos índices de desempenho que o controle do PIDPlus, manteve o mesmo patamar de desempenho do caso ideal (sem falhas), mesmo com as falhas inseridas.

TABELA I

DESEMPENHO DOS CONTROLADORES COM FALHAS DE COMUNICAÇÃO

\begin{tabular}{|c|c|c|}
\hline \multirow{2}{*}{$\begin{array}{c}\text { Índice de } \\
\text { Desempenho }\end{array}$} & \multicolumn{2}{|c|}{ Controle } \\
\cline { 2 - 3 } & PID Tradicional + Falha & PIDPlus + Falha \\
\hline IAE sem Falha & 38 & 26 \\
\hline ITAE sem Falha & 3419 & 2903 \\
\hline IAE (60x5) & 54 & 29 \\
\hline ITAE (60x5) & 7098 & 3071 \\
\hline IAE (30x6) & 95 & 36 \\
\hline ITAE (30x6) & 14033 & 4266 \\
\hline IAE (15x6) & 144 & 32 \\
\hline ITAE (15x6) & 22640 & 3480 \\
\hline IAE Falha Aleatória & --- & 29 \\
\hline $\begin{array}{c}\text { ITAE Falha } \\
\text { Aleatória }\end{array}$ & --- & 3049 \\
\hline
\end{tabular}


Os resultados das Fig. 11, Fig. 12 e da Tabela II mostram que o controlador PIDPlus apresenta melhor robustez, controlando o WNCS com melhor desempenho em todos os casos de presença de falhas de comunicação. O controlador PIDPlus mantém o último valor da saída do controlador (sinal de controle), pois ele calcula as contribuições do termo integral e derivativo para a saída do controlador somente quando há uma atualização da medição da variável e usa o tempo decorrido entre as atualizações em seus cálculos. Assim, o PIDPlus atua apenas quando é recebido um novo valor da medição e considera que a mudança na variável medida ocorreu não apenas no último período de execução do controlador, mas ao longo do tempo decorrido entre as atualizações. Dessa forma, quando há uma perda de comunicação com o transmissor ou elemento final de controle, o PIDPlus não fornece outra ação de reposição, ele aguarda novas atualizações. Quando a comunicação é restabelecida, ele atua sobre a nova atualização.

Nos experimentos anteriores, o controlador do WNCS estava operando com um período de amostragem do sensor sem fio (intervalo entre transmissões de mensagem da variável controlada) de $2 \mathrm{~s}$. Em WNCS, o período de amostragem do sensor pode influenciar o desempenho de controle do sistema, além de ser um parâmetro diretamente relacionado ao consumo energético do sensor sem fio [14]. Do ponto de vista energético, quanto maior o período de amostragem do sensor, sem prejuízo do desempenho de controle, melhor para o WNCS. Dessa forma, é importante conhecer a influência deste parâmetro no desempenho do WNCS e o período de amostragem limite para obtenção de um desempenho aceitável para o WNCS operando com o PIDPlus. Para a realização destas análises, o período de amostragem do sensor sem fio foi variado de 2 até $20 \mathrm{~s}$. A comparação das respostas do WNCS é mostrada na Fig. 13.

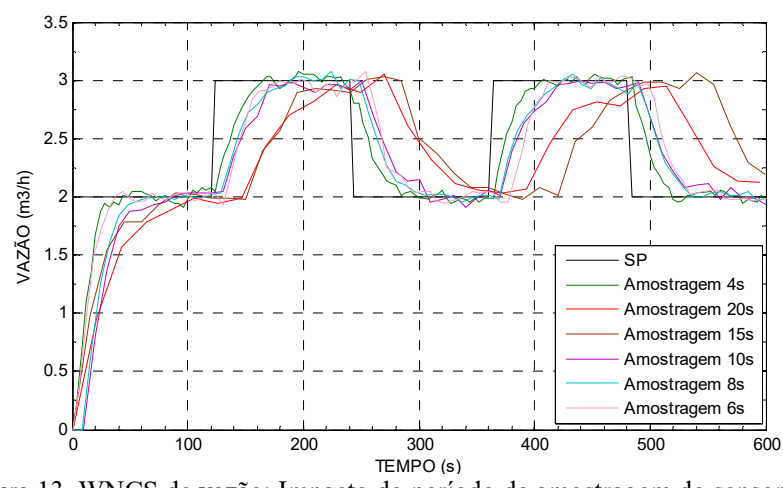

Figura 13. WNCS de vazão: Impacto do período de amostragem do sensor sem fio na resposta.

Nota-se na Fig. 13 que para alterações do período de amostragem de até $10 \mathrm{~s}$, o controlador PIDPlus conseguiu manter uma resposta adequada ao perfil do setpoint. No entanto, para valores acima de $10 \mathrm{~s}$, o controle apresenta queda de desempenho, sendo este o período de amostragem limite para obtenção de um desempenho aceitável para o WNCS. Essa é uma constatação importante pois ao contrário do PIDPlus, o PID tradicional, que é discretizado e sintonizado para um período de amostragem específico (2s) tem seu desempenho degradado [15]. Isso confirma uma das características do PIDPlus: não é necessária uma nova sintonização dos ganhos do controlador PIDPlus, ou seja, o controlador PIDPlus pode ser projetado a partir do PID tradicional.

O último parâmetro analisado em relação ao seu impacto no desempenho de controle do WNCS desenvolvido foi o atraso de comunicação (delay). Em WNCS, o atraso de comunicação total (atraso do sensor -controlador + atraso controlador - atuador) geralmente é variável no tempo. Para a realização desta análise, foi inserido no loop de controle do WNCS um intervalo de tempo randômico, de forma a representar a característica variável de um atraso de comunicação. Esse atraso foi variado aleatoriamente em três casos diferentes: atraso de até $2 \mathrm{~s}$, de até $5 \mathrm{~s}$ e de até $10 \mathrm{~s}$. A comparação das respostas do WNCS é mostrada na Fig. 14.

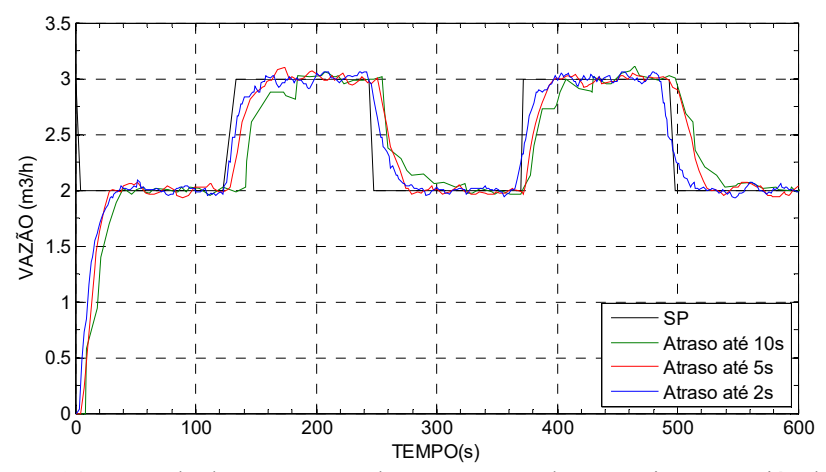

Figura 14. Controle de vazão: PIDPlus com atraso de comunicação variável.

Os resultados a Fig. 14 mostram que o controlador PIDPlus consegue operar adequadamente na presença de atrasos variantes no tempo. Nos testes realizados com diferentes valores de atrasos de comunicação inseridos na malha de controle fechado, o desempenho do WNCS se manteve próximo ao ideal (caso inicial onde não foi inserido atraso adicional).

\section{CONCLUSÃO}

Os resultados demonstram que a implementação em hardware dedicado do protocolo Modbus para a comunicação via TCP/IP e Wi-Fi foram efetivas, permitindo adaptar instrumentos analógicos em dispositivos Modbus e transmitir sinais de controle e realimentação de forma confiável. Além disso, o código gerado pela implementação da camada de aplicação do Modbus na plataforma Arduino, permite reuso em outras plataformas de hardware embarcado, tornando-as compatíveis com aplicações Modbus.

O controlador PIDPlus atende ao controle de processos que utilizam transmissores com e sem fio, mostrando-se com melhor robustez e desempenho superior ao PID tradicional nos casos estudados de perda de dados transmitidos, presença de atrasos de comunicação variantes no tempo e alteração o período de amostragem do sensor sem fio. No caso de perda de dados transmitidos ou falhas de comunicação, o PIDPlus foi capaz de manter desempenho equiparável ao caso ideal (sem falhas) para os casos de falhas sequenciais e aleatórias. Também se constatou que controlador PIDPlus opera adequadamente em uma faixa de período de amostragem que inclui a frequência de sintonia do PID tradicional e períodos de amostragem variáveis, com a vantagem de não requerer nova sintonia do controlador. 
Adicionalmente, o uso do controlador PIDPlus para WNCS é fortemente recomendado nas seguintes situações: o tempo de atualização da malha de controle fechado é maior do que o tempo de resposta do processo; aplicações com maior probabilidade de ocorrência de erros e falhas de comunicação na rede sem fio;

elemento final de controle apresenta significativa manutenção em função dos grandes picos de acionamento, fazendo com que o equipamento trabalhe em seu limite projetado.

\section{AGRADECIMENTOS}

Os autores agradecem ao apoio para este trabalho: 2015/01303-2, Fundação de Amparo à Pesquisa do Estado de São Paulo (FAPESP).

\section{REFERÊNCIAS}

[1] B. Galloway and G.P Hancke, "Introduction to Industrial Control Networks", IEEE Comm. Surveys \& Tutorials, vol.15, pp. 860-880, 2013.

[2] T. Sauter, "The Three Generations of Field-Level Networks-Evolution and Compatibility Issues", IEEE Trans. Industrial Electronics, vol. 57, no. 11, pp. 3585-3595, Nov, 2010.

[3] R.A. Gupta and M.Y. Chow, "Networked Control System: Overview and Research Trends", IEEE Trans. Industrial Electronics", vol. 57, no. 7, pp. 2527-2535, Nov, 2010.

[4] J. Baillieul and P.J. Antsaklis, "Control and Communication Challenges in Networked Real Time Systems", Proc. IEEE Tech. of Networked Control Systems, vol. 95, pp. 09-28, 2007.

[5] C. Fischione, P. Park, P. Di Marco and K.H. Johansson, "Design Principles of Wireless Sensor Networks Protocols for Control Applications", S.K. Mazumder (Ed.). Wireless Networking Based Control, Ch. 9, Springer, pp. 203-238, 2011.

[6] M. Paavola and K. Leiviska, "Wireless Sensor Networks in Industrial Automation", J.S. Blanes (Ed.). Factory Automation, Ch 10, Intech, pp. 201220, 2010.

[7] S. Petersen and S. Carlsen, "Wireless HART Versus ISA100.11a: The Format War Hits the Factory Floor", IEEE Industrial Electronics Magazine, $\mathrm{v}$ vol. 5, no.4, pp. 23-34, 2011.

[8] P. Naghshtabrizi and J.P. Hespanha, "Implementation Considerations for Wireless Networked Control Systems", S.K. Mazumder (ed.), Wireless Networking Based Control, Ch. 1, Springer, pp. 1-27, 2011.

[9] T. Blevins, M. Nixon and W. Wojsznis, "PID Control Using Wireless Measurements", in American Control Conference (ACC), pp. 1-6, 2014.

[10] Modbus, 2015. “Modbus Organization”. Disponível em: http://www.modbus.org. Acesso em: Dezembro, 2015.

[11] Y.A. Millan, F. Vargas, F. Molano and E. Mojica, "A Wireless Networked Control Systems Review", in IEEE IX Latin American and IEEE Colombian Conference on Automatic Control and Industry Applications, 2011.

[12] J. Song, A.K. Mok, D. Chen, M. Nixon, T. Blevins and W. Wojsznis, "Improving PID control with unreliable communications", in ISA EXPO Technical Conference, 2006.

[13] D.M. Anand, J.R Moyne and D.M Tilbury, "Performance evaluation of wireless networks for factory automation applications", in 5th Annual IEEE Conf. on Automation Science and Engineering (CASE), Bangalore, India, pp. 340-346, August 22-25, 2009.

[14] R.K. Mansano, E.P. Godoy and A.J.V. Porto, "The Benefits of Soft Sensor and Multi-rate Control for the Implementation of Wireless Networked Control Systems", Sensors, pp. 24441-24461, 2014.

[15] Y.S. Andrade, R.K. Mansano and E.P. Godoy, "Projeto de Controle Não Periódico para Sistemas de Controle Via Redes Sem Fio", in Congresso Brasileiro de Automática (CBA), Vitória, Brasil, 2016.

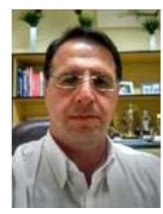

Marcos Rogério Caldiéri received the B.Sc. degree in Physics in 2002, the Academic specialization in Mechatronics Engineering in 2007 and the M.Sc. in Electrical Engineering in 2016, all from the São Paulo State University (UNESP) at Bauru (SP-Brazil). Currently he is the Technical Coordinator of Courses of the National Service for Industrial Learning (SENAI) at Lençóis Paulista (SP-

Brazil). His research interests include simulators and practical applications of networked control systems (NCS) and control theory. http://lattes.cnpq.br/9903703582966153.

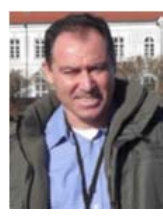

Jeferson André Bigheti received the B.Tech. degree in Electrical Systems in 1990, the Academic specialization in Mechatronics Engineering in 2007 and the M.Sc. in Electrical Engineering in 2011, all from the São Paulo State University (UNESP) at Bauru (SP-Brazil). He also has a major in Special Program of Pedagogical Training for Profissional Education at University of Southern Santa Catarina (UNISUL) in 2005. Currently he is a Ph.D student in Electrical Engineering and a professor of the National Service for Industrial Learning (SENAI) at Lençóis Paulista (SP-Brazil). His research interests include manufacturing automation, robotics, instrumentation, industrial networks and communication protocols, supervision of industrial systems, networked control systems (NCS), wireless applications, Internet of Things, Industry 4.0 and embedded electronics. http://lattes.cnpq.br/6243932250481498.

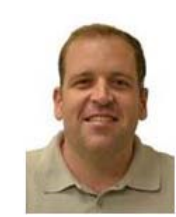

Eduardo Paciencia Godoy received the B.Eng. degree in Control and Automation Engineering at Itajubá Federal University (MG-Brazil) in 2003 and the M.Sc. and Ph.D. degrees in Mechanical Engineering at University of São Paulo at São Carlos (SP-Brazil) in 2007 and 2011, respectively. Currently he is an Assistant Professor of the São Paulo State University (UNESP) at Sorocaba (SP-Brazil). His research interests include industrial networks and automation, networked control systems (NCS), wireless networks and telemetry, embedded electronics, Internet of Things and Industry 4.0. http://lattes.cnpq.br/0072632067545698. 